\title{
Possible experimental test of the nonlinear phononics interpretation of light-induced superconductivity
}

\author{
M. Altarelli ${ }^{*}{ }^{*}$ \\ Max Planck Institute for Structure and Dynamics of Matter, 22761 Hamburg, Germany
}

(Received 12 March 2020; revised 20 August 2020; accepted 21 August 2020; published 9 September 2020)

\begin{abstract}
Experimental evidence for a transient enhancement of the superconducting critical temperature of $\mathrm{YBa}_{2} \mathrm{Cu}_{3} \mathrm{O}_{6+x}$ in the presence of an intense $\mathrm{THz}$ or infrared pump pulse was ascribed to nonlinear phononics effects. Here, I introduce a simple phenomenological Ginzburg-Landau model of this phenomenon, to explore further consequences and possible experimental tests of this interpretation. This treatment predicts that, upon cooling below $T_{c}$ in the absence of pumping, (a) an abrupt softening of a Raman-active mode frequency and (b) a spontaneous lattice distortion, growing linearly with $\left(T_{c}-T\right)$ should occur. Numerical estimates for $\mathrm{YBa}_{2} \mathrm{Cu}_{3} \mathrm{O}_{6+x}$ indicate that the frequency softening could likely be observable, whereas the lattice distortion may be too small. A comparison with Raman experiments for the relevant phonon modes in $\mathrm{YBa}_{2} \mathrm{Cu}_{3} \mathrm{O}_{6+x}$ does not lend support to the nonlinear phononics interpretation. On the other hand, however, a very large (up to $18 \%$ ) phonon frequency softening just below $T_{c}$, qualitatively similar to the predictions of the present model for its size and abrupt temperature dependence, was observed over 20 years ago in $\mathrm{HgBa}_{2} \mathrm{Ca}_{3} \mathrm{Cu}_{4} \mathrm{O}_{10+x}$; its explanation in terms of Josephson plasmons has been controversial. Light-induced superconductivity still needs to be investigated in this material and it may be of interest to explore if it is present and possibly connected, via the mechanism discussed here, to the observed anomalous phonon behavior.
\end{abstract}

DOI: 10.1103/PhysRevResearch.2.033384

\section{INTRODUCTION}

The observation of the phenomenon termed light-enhanced or light-induced superconductivity [1-4] has raised large interest. In the presence of an intense sub-ps $\mathrm{THz}$ or infrared (IR) pump pulse, the optical properties of materials such as $\mathrm{YBa}_{2} \mathrm{Cu}_{3} \mathrm{O}_{6+x}$ and $\mathrm{K}_{3} \mathrm{C}_{60}$, when probed at temperatures well above their respective superconducting critical temperatures, showed dynamical conductivity spectra very similar to those of the superconducting phase. This evidence for a transient radiation-induced phase, with unprecedented superconducting critical temperatures, up to room temperatures, has stimulated theoretical and experimental work, aimed at providing and testing an explanation of this remarkable phenomenon. Among these efforts, the nonlinear lattice dynamics, or nonlinear phononics explanation [5-7], of light-enhanced superconductivity gained the most attention, especially after the ultrafast X-ray experiments by Mankowsky et al. [7].

According to this explanation, the most important effect of the pump pulse is to push one (or more) optical phonon $A_{1 g}$-symmetric mode coordinate $Q$ off its equilibrium value by excitation of an infrared-active mode $Q_{\mathrm{IR}}$, due to a nonlinear

\footnotetext{
*massimo.altarelli@mpsd.mpg.de
}

Published by the American Physical Society under the terms of the Creative Commons Attribution 4.0 International license. Further distribution of this work must maintain attribution to the author(s) and the published article's title, journal citation, and DOI. Open access publication funded by the Max Planck Society. coupling that, according to Mankowsky et al.'s [7] arguments, is of the form $-a_{1,2} Q Q_{\mathrm{IR}}^{2}$, where the suffix 1 (2) of the coupling constant refers to the linear (quadratic) dependence on $Q$ $\left(Q_{\mathrm{IR}}\right)$; here, and in the following, the $\mathbf{k}$ dependence of phonon modes is not mentioned explicitly, as by optical excitation only modes at the $\Gamma$ point are being excited. Indeed, when this term is added to the harmonic potentials and the kinetic energies of the $Q_{\mathrm{IR}}, Q$ oscillators, the Barbanis Hamiltonian [8] is obtained, which has been thoroughly investigated in the context of celestial mechanics, and in studies of quantum ergodicity and in molecular physics [9-11]; it turns out that an accurate approximation to the exact eigenfunctions of this Hamiltonian is given by eigenfunctions of the form $[10,11]$

$$
\Phi_{n_{1}, n_{2}}=N \phi_{n_{1}}(a Q+b) \phi_{n_{2}}\left(a_{\mathrm{IR}} Q_{\mathrm{IR}}\right),
$$

where $N$ is a normalization constant, and the $\phi$ are harmonic oscillator eigenfunctions for scaled oscillator coordinates, and, for the coordinate $Q$, with a displaced oscillator coordinate; the scaling and displacement terms $a, a_{\mathrm{IR}}$ and $b$ can be determined variationally or within a self-consistent field (SCF) approach for a given value of $a_{1,2}$. These approximate results are supported by state-of-the-art $a b$ initio density functional theory (DFT) calculations [12] for $\mathrm{YBa}_{2} \mathrm{Cu}_{3} \mathrm{O}_{6+x}$ (YBCO), which also support the assumption that the thirdorder Barbanis-type anharmonicity dominates over quartic and higher terms for the regime of pump intensities in the relevant experiments.

With the $Q$ lattice coordinate pushed to an off-equilibrium value by the excitation of the infrared mode by a pump pulse, a transient modified crystal structure sets in, and this could 
explain the substantial transient increase of $T_{c}$ [1-3]. Pumpprobe $\mathrm{x}$-ray diffraction experiments on a sub-ps timescale support this interpretation [7]. A more recent experimental and theoretical analysis of the pump-frequency dependence over the complete far- to near-IR range [13] definitely identified the $670 \mathrm{~cm}^{-1} B_{1 u}$ infrared-active mode as by far the most effective in generating the higher-temperature superconductinglike response; but, on the other hand, it raised some serious questions, based on ab initio DFT lattice dynamics calculations, about the identification of the involved $A_{1 g}$-symmetric phonon mode(s).

It is therefore worthwhile to look for possible further experimental validations of the nonlinear phononics interpretation of light-induced superconductivity, an important attempt to understand transient light-induced phases, and this is the purpose of the present paper. It seems indeed hardly plausible that an interplay between vibrational/structural and electronic properties that is so strong as to induce a raise of the superconducting temperature by hundreds of degrees does not affect any other observable quantities.

If a given phonon coordinate affects the electronic states at the Fermi energy and considerably modifies $T_{c}$, as argued in Ref. [7], one could suspect that changes of the electronic states at the Fermi energy, such as the usual onset of equilibrium superconductivity when cooling below $T_{c}$, with the opening of a gap, could have effects on the phononic properties, going beyond the ordinary small corrections to the phonon selfenergies $[14,15]$. This paper investigates the possibility that this could lead to observable effects that could provide support for the nonlinear phononics interpretation of light-induced superconductivity. If the predicted effects are not observed, on the other hand, this is not necessarily ruling out this interpretation, as discussed later.

It would be beyond the scope of this paper to suggest modifications or extensions of the nonlinear phononics explanation as formulated in Refs. [5-7], and in the following we shall not question the assumptions adopted there, for example, ignoring the effects of the pump pulse on the population of electronic states, or retaining only the Barbanis type of anharmonic coupling to a single phonon mode and ignoring other possible terms, such as the cubic and fourthorder terms involving modes off the zone center discussed by Knap et al. [16].

For this admittedly limited purpose, we adopt a very simple-minded Ginzburg-Landau description of the superconducting transition [17], including an additional dependence of the critical temperature $T_{c}$ on the $Q$ lattice coordinate. Reasoning based on this phenomenological description implies that, even in the absence of the pump, when the system is cooled below $T_{c}$, a softening of this phonon frequency and a spontaneous distortion of the lattice should occur. An attempt to estimate the sizes of the softening and of the distortion is carried out, at least roughly, to determine whether they could be observable; measured experimental properties of $\mathrm{YBa}_{2} \mathrm{Cu}_{3} \mathrm{O}_{6+x}$ are used to infer the values of the parameters entering the Ginzburg-Landau free energy. It turns out that, for plausible values of the parameters, the frequency shift should be observable, whereas the lattice distortion could be harder to detect.

\section{GINZBURG-LANDAU FUNCTIONAL FOR THE FREE ENERGY}

In the phenomenological Ginzburg-Landau theory, the free energy per unit volume of a superconductor in the vicinity of $T_{c}$ is written as a functional of the order parameter $\Psi$; in the simplest case of a uniform superconductor without external fields, it takes the form

$$
F(T,|\Psi|)=a\left(T-T_{c}\right)|\Psi|^{2}+b|\Psi|^{4},
$$

where the coefficients $a, b$ are positive. Looking for a minimum of this functional as a function of $|\Psi|$, one immediately finds $|\Psi|=0$ for $T>T_{c}$, and for $T<T_{c}$ the values

$$
|\Psi|^{2}=\frac{a\left(T_{c}-T\right)}{2 b}, \quad F_{\min }(T)=\frac{-a^{2}\left(T_{c}-T\right)^{2}}{4 b} .
$$

It can be argued that this mean-field type of formulation is oversimplified for the cuprates, with their short coherence length, their symmetry lower than cubic, etc.. But for the sake of simplicity we shall stick to this description. The observation of similar light-induced transient superconductivity phenomena in totally different compounds, such as $\mathrm{K}_{3} \mathrm{C}_{60}$ [4], supports a rather generic approach. We further assume that an optical phonon coordinate $Q$, with a vanishing expectation value in the equilibrium state above $T_{c}$, is affecting the transition temperature, if it takes a nonvanishing amplitude, and that this is what happens when an infrared-active phonon $Q_{\mathrm{IR}}$ is pumped by a sufficiently intense laser pulse. Right after the pulse, the new equilibrium position is $Q_{0}$, proportional to $Q_{\mathrm{IR}}^{2}$. A simple description of the effect on the superconducting critical temperature of this new transient structure can be given by modifying the free-energy functional in the following way,

$$
\begin{aligned}
F\left(T,|\Psi|, Q_{0}\right)= & E_{0}+(1 / 2) \rho k\left(Q-Q_{0}\right)^{2} \\
& +a\left[T-T_{c}-\Theta_{c}(Q)\right]|\Psi|^{2}+b|\Psi|^{4} .
\end{aligned}
$$

Here, the first two terms on the right-hand side correspond to the elastic energy (per unit volume) of the lattice distortion around the new equilibrium position, where $E_{0}<0$ is the bottom of the harmonic potential well at $Q_{0}, \rho$ is the number density of deformed bonds, and $k=m^{*} \omega^{2}$ the product of effective mass and square frequency. The third term expresses an increase of the critical temperature to $T_{c}+\Theta_{c}(Q)$, when $Q$ is nonvanishing (i.e., when it is displaced with respect to the equilibrium position in the absence of pumping, not from $Q_{0}$ ). The function $\Theta_{c}(Q)$ reflects the complex physics that leads to a $T_{c}$ enhancement in a distorted lattice. According to the arguments in Refs. [5,6], the lattice distortion induces a modification of the orbital character and density of electronic states at the Fermi energy, leading to the $T_{c}$ enhancement. Lacking a microscopic theory, the functional relationship between $Q$ and $T_{c}$ remains out of our reach. Once again, a word of caution on the questionable aspects of this approach is necessary. As it was stated above, the Ginzburg-Landau theory is limited to $T$ near $T_{c}$. Just shifting the critical temperature keeping the same functional form and $a, b$ parameters is really justified if the shift satisfies

$$
\Theta_{c}(Q) \ll T_{c} .
$$

This restriction certainly does not apply to the situation described in Refs. [3,13] which implies, e.g., a shift of some 
$250 \mathrm{~K}$ from $50 \mathrm{~K}$ to room temperature for the critical temperature of $\mathrm{YBa}_{2} \mathrm{Cu}_{3} \mathrm{O}_{6.5}$ after a $\mathrm{THz}$ pulse. So one must understand Eq. (4) as describing the "small $Q$ " regime, where Eq. (5) is satisfied, and not the regime of intense THz pumping that the experiments access. In fact, as we shall soon see, the conclusions of this paper are for the case in which the pumping of $Q_{\mathrm{IR}}$ is absent.

In order to make progress we make a linearization assumption for $\Theta_{c}(Q)$, i.e., assume that in some limited range of $Q$ near $Q=0$,

$$
\Theta_{c}(Q) \simeq c Q
$$

[remember that $\Theta_{c}(Q=0)=0$ ], where $c$ is a coefficient to be determined. This is very likely the case for a sufficiently small $Q$ range [the exception being if, for some reason, $\left(\partial \Theta_{c} / \partial Q\right)_{Q=0}$ vanishes, and the small- $Q$ development starts with a $Q^{2}$ term; however, if the lattice structure modification corresponding to $Q$ enhances $T_{c}$, it seems highly improbable that this effect is an even function of $Q$ ]. Within this assumption Eq. (4) becomes

$$
\begin{aligned}
F(T,|\Psi|, Q)= & E_{0}+(1 / 2) \rho k\left(Q-Q_{0}\right)^{2} \\
& +a\left[T-T_{c}-c Q\right]|\Psi|^{2}+b|\Psi|^{4} .
\end{aligned}
$$

Additional support for this form of the free-energy functional comes from the discussion of the coupling of the strain tensor to the superconducting order parameter by Joynt and Rice [18], Ozaki [19], and Millis and Rabe [20] who, starting from a simple description of the condensation energy in terms of the band structure and the gap function, suggest coupling terms linear in the strain and quadratic in the order parameter (for a summary and extensive information on a generalization of the argument for anisotropic superconductors, see Ref. [21]). This is exactly the character of the additional term in Eq. (7), $-c Q|\Psi|^{2}$. Although the strain tensor is representative of acoustical deformations, and here $Q$ represents an optical deformation, this should not modify the argument.

If a certain value of $Q_{0} \neq 0$ is imposed by the external $\mathrm{THz}$ pump, we can minimize the free-energy Eq. (7) with respect to both $|\Psi|^{2}$ and $Q$ to get

$$
\begin{aligned}
|\Psi|_{\text {min }}^{2} & =\rho k \frac{a\left(T_{c}+c Q_{0}-T\right)}{2 b \rho k-a^{2} c^{2}}, \\
Q_{\text {min }} & =Q_{0}+\frac{a^{2} c\left(T_{c}+c Q_{0}-T\right)}{2 b \rho k-a^{2} c^{2}},
\end{aligned}
$$

and

$$
F_{\min }\left(T, Q_{0}\right)=E_{0}-\frac{1}{2} \rho k a^{2} \frac{\left(T_{c}+c Q_{0}-T\right)^{2}}{2 b \rho k-a^{2} c^{2}} .
$$

For Eqs. (8) and (9) to make sense, the conditions $T_{c}+c Q_{0}>$ $T$ (necessary for the existence of a minimum) and $2 b \rho k>$ $a^{2} c^{2}$ (necessary for a positive $|\Psi|^{2}$ ) must be fulfilled. The latter restricts the admissible values of $c$ to the range

$$
c^{2}<c_{\max }^{2} \equiv \frac{2 b \rho k}{a^{2}} .
$$

\section{CONSEQUENCES IN THE ABSENCE OF AN EXTERNAL PUMP PULSE}

In this section we shall consider the situation in which no external pump is present, so that $Q_{0}=0$ (and of course $E_{0}=$ $0)$. Note that, if the critical temperature is enhanced by a lattice distortion $(c \neq 0)$, Eq. (8) shows that $Q_{\min }$ does not vanish even when $Q_{0}$ does:

$$
Q_{\min }=\frac{a^{2} c\left(T_{c}-T\right)}{2 b \rho k-a^{2} c^{2}} \equiv \frac{c\left(T_{c}-T\right)}{c_{\max }^{2}-c^{2}} .
$$

It is therefore easy to see that a spontaneous distortion of the lattice, proportional to $T_{c}-T$, takes place below $T_{c}$, because

$$
F_{\min }\left(T, Q_{0}=0\right)=-\frac{1}{2} \rho k a^{2} \frac{\left(T_{c}-T\right)^{2}}{2 b \rho k-a^{2} c^{2}}
$$

lies deeper than the corresponding minimum of the undistorted lattice, given by Eq. (3),

$$
-\frac{1}{2} \rho k a^{2} \frac{\left(T_{c}-T\right)^{2}}{2 b \rho k-a^{2} c^{2}}<\frac{-a^{2}\left(T_{c}-T\right)^{2}}{4 b}
$$

as the inequality, in the range of validity of Eq. (10), reduces to the obvious one:

$$
\frac{2 b \rho k}{2 b \rho k-a^{2} c^{2}}=\frac{c_{\max }^{2}}{c_{\max }^{2}-c^{2}}>1 .
$$

Under the same no-pump conditions, consider now the change in the free energy, given by Eq. (7) with $Q_{0}=0$, when we excite the vibrational mode slightly away from the minimum value given by Eq. (11). For a small deviation $\Delta Q$ the free energy is

$$
\begin{aligned}
F\left(T, Q=Q_{\min }+\Delta Q\right)= & F_{\min }\left(T, Q_{\min }\right) \\
& +(1 / 2)\left(\rho k-\frac{a^{2} c^{2}}{2 b}\right) \Delta Q^{2} .
\end{aligned}
$$

In deriving Eq. (15) we assume that the electronic properties, including the superconducting order parameter, instantaneously adjust to the lattice configuration, and therefore minimize the free energy with respect to $|\Psi|^{2}$ for the value of $Q=Q_{\min }+\Delta Q$. This assumption appears questionable, as the energy scale of electronic excitations (essentially the superconducting gap) is certainly not larger than the scale of the relevant Raman-active phonon energies, typically $\simeq 30-40 \mathrm{meV}$, and does not straightforwardly justify a Born-Oppenheimer approach. Nonetheless, there are good experimental and theoretical reasons to mitigate the severity of this assumption. First of all, the main experimental effect in discussion here is the emergence of a fully developed superconductinglike optical response $0.5 \mathrm{ps}$ [13] or $0.8 \mathrm{ps}$ after the pump pulse [3]. This implies a dramatic gap opening (buildup of the order parameter) on a timescale faster than $0.5-0.8 \mathrm{ps}$, in a range of temperatures down to $T_{c}$. If this very fast response of the order parameter were not possible, then the whole observation of light-enhanced superconductivity would be in question. A timescale faster than $0.5-0.8$ ps can still be slower than the phonon periods of order $0.1 \mathrm{ps}$, but suggests that response times cannot be simply related to one energy scale. In the special case of cuprates, for example, the anisotropy of the $d$-wave gap, displaying nodal directions, 
was shown in model calculations [22] to lead to a much faster dynamic response of the superconducting gap to sudden perturbations, with respect to the isotropic $s$-wave case. Although the anisotropy is ignored in our simple description, the resulting ultrafast response time can be phenomenologically incorporated. For these reasons, rather than attempting a more realistic but more complex approach taking the details of the time-dependent response into account, we adopt the simplifying assumption of an instantaneous relaxation of the superconducting order parameter.

The result of Eq. (15) corresponds to a reduction of the restoring force constant, or, equivalently, to a softening of the bare phonon frequency $\omega$ as a function of $c$ in the form

$$
\omega_{\mathrm{soft}}^{2}=\omega^{2}\left(1-\frac{c^{2}}{c_{\max }^{2}}\right) .
$$

It is important to remark that the softening described by Eq. (16) takes place abruptly, as soon as $T$ is lowered below the critical temperature.

We therefore argued so far that the $Q$ dependence of the effective critical temperature implies two consequences: (1) a softening of the phonon frequency (reduction of the "spring constant") taking place below $T_{c}$ in agreement with Eqs. (15) and (16), and (2) a spontaneous distortion from $Q=0$ to $Q_{\min }(T)$ given by Eq. (11), linearly increasing in size as the temperature is lowered below $T_{c}$.

Questions to address are whether the simple-minded treatment adopted here can lead to credible predictions, and whether the size of the predicted effects is possibly leading to observable effects. In order to explore the latter, one needs to establish numerical values of the model parameters in a realistic case.

\section{NUMERICAL ESTIMATES FOR YBCO}

According to Ref. [7], the main effect of the THz pumping pulse is to generate a nonequilibrium elongation of the intrabilayer distance, via nonlinear phonon couplings. This elongation corresponds (see Ref. [7], Extended Data Fig. 3) to the modes labeled $A_{g} 15$ and $A_{g} 21$, with wave numbers 94.52 and $125.19 \mathrm{~cm}^{-1}$, respectively. This assignment, based on ultrafast x-ray diffraction data, is, however, not supported by theory, as more recent work by Fechner and Spaldin [12] and Liu et al. [13], based on DFT calculations, suggests that the relevant $A_{1 g}$ mode has a substantially higher frequency, $470 \mathrm{~cm}^{-1}$ or more. Nonetheless, just to get an order of magnitude estimate, let us take Mankowsky et al.'s estimate and further simplify this to a single mode; to evaluate the parameter $k=m^{*} \omega^{2}$, let us assume a wave number of $\simeq 100 \mathrm{~cm}^{-1}$, corresponding to $\omega \simeq 2 \times 10^{13} \mathrm{~s}^{-1}$. The effective mass corresponding to the relative coordinate of two $\mathrm{Cu}$ atoms is $(1 / 2) m_{\mathrm{Cu}} \simeq(1 / 2) 63.5 \mathrm{amu}$, where $1 \mathrm{amu}=1.66 \times 10^{-24} \mathrm{~g}$ and 63.5 is the isotope-averaged mass of a $\mathrm{Cu}$ atom. One thus comes to

$$
k \simeq 2.1 \times 10^{4} \mathrm{~g} / \mathrm{s}^{2} .
$$

[Note that this purely indicative estimate of $m^{*} \omega^{2}$ is unchanged for a different, O-related phonon mode, if the effective mass is reduced by a factor 4 (from $\mathrm{Cu}$ to $\mathrm{O}$ ) and the frequency is increased by a factor of 2.]

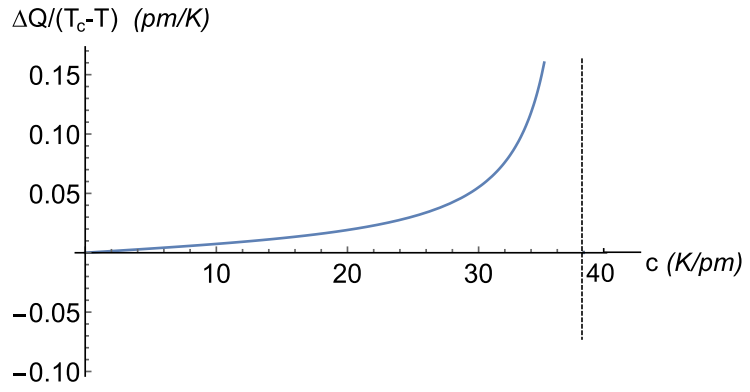

FIG. 1. Lattice distortion in pm, divided by $T_{c}-T$ in $\mathrm{K}$, as a function of the linearization parameter $\mathrm{c}$, in $\mathrm{K} / \mathrm{pm}$. The vertical dashed line marks the $c_{\max }$ value, $38 \mathrm{~K} / \mathrm{pm}$.

The density of $\mathrm{Cu}$ pairs facing each other across a bilayer in the ortho-II structure of YBCO is one pair in a volume corresponding to $3.827 \times 3.893 \times 11.699 \AA^{3}[23]$ that establishes another parameter,

$$
\rho \simeq 5.7 \times 10^{21} \mathrm{~cm}^{-3} .
$$

In order to obtain the value of the parameter $a^{2} / 2 b$, which determines (together with the linearization coefficient $c$ ) the phonon softening, Eq. (16), and the lattice distortion, Eq. (11), we use a relation involving the specific heat jump per unit volume at $T_{c}$ [see Eq. (45.8) in Ref. [17]],

$$
(1 / V)\left(C_{s}-C_{n}\right)=\frac{a^{2} T_{c}}{2 b},
$$

where $C_{s}\left(C_{n}\right)$ denotes the specific heat at $T_{c}$ of the superconducting (normal) phase.

Experimental determinations of the specific heat jump at $T_{c}$ are found in the literature. For $\mathrm{YBa}_{2} \mathrm{Cu}_{3} \mathrm{O}_{6.5}$, Loram et al. [24] obtain for $\Delta \gamma=\gamma_{s}-\gamma_{n}$, where $\gamma=C / T$,

$$
\Delta \gamma\left(T_{c}\right) \simeq 6.2 \frac{\mathrm{mJ}}{\mathrm{mol} \mathrm{K}^{2}},
$$

whereas Wühl et al. [25] measure

$$
\Delta \gamma\left(T_{c}\right) \simeq 10 \frac{\mathrm{mJ}}{\mathrm{mol} \mathrm{K}^{2}} .
$$

So an average of the experimental results gives an estimate of $\simeq 8 \mathrm{~mJ} /\left(\mathrm{mol} \mathrm{K}^{2}\right)$. The critical temperature of $\mathrm{YBa}_{2} \mathrm{Cu}_{3} \mathrm{O}_{6.5}$ is about $50 \mathrm{~K}$, and the density of the material (necessary to estimate the molar volume) is $6.3 \mathrm{~g} / \mathrm{cm}^{3}$ [26] and we find

$$
\frac{C_{s}-C_{n}}{V T_{c}}=\frac{a^{2}}{2 b}=\frac{8.5 \times 10^{-5} \mathrm{~J}}{\mathrm{~cm}^{3} \mathrm{~K}^{2}} .
$$

Now all the ingredients are there to determine the numerical value of $c_{\max }$. One obtains

$$
c_{\max }^{2}=\frac{2 b \rho k}{a^{2}} \simeq 1.4 \times 10^{23} \mathrm{~K}^{2} / \mathrm{cm}^{2},
$$

or $c_{\max } \simeq 3.8 \times 10^{11} \mathrm{~K} / \mathrm{cm}$. With the help of Eqs. (11) and (15), we can now plot the lattice distortion and the softening of the frequency as a function of the linearization parameter $c$ (Figs. 1 and 2). 


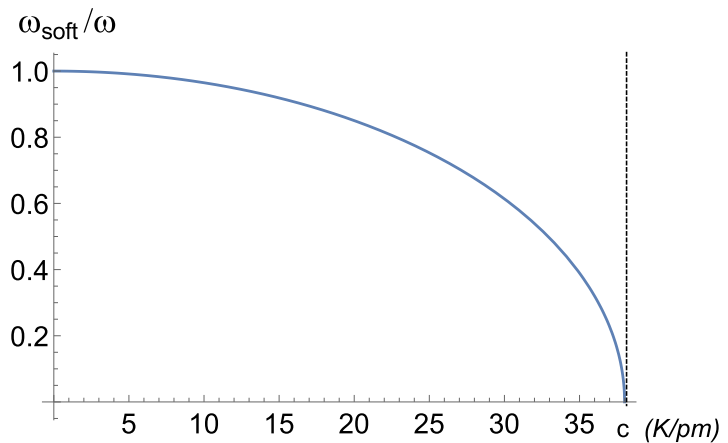

FIG. 2. Relative softening of the phonon frequency as a function of $c$.

\section{COMPARISON WITH EXPERIMENT}

The question whether these are observable effects is difficult to answer in the absence of a reasonable way to estimate the parameter $c$. Nonetheless, with a resolution between 1 and $3 \mathrm{~cm}^{-1}$ for Raman experiments [27], the softening should be easily detectable as soon as $c$ exceeds $\simeq 0.2 c_{\max }$, especially because of its sudden onset as the temperature is lowered below $T_{c}$. The lattice distortion, which grows linearly with $T_{c}-T$, on the other hand, would be limited to $0.2 \mathrm{pm}$ at $10 \mathrm{~K}$ below $T_{c}$, which is may be below the detection limit (one should not feel comfortable about considering still lower temperatures, as this would drive us outside of the region where Ginzburg-Landau arguments can possibly apply).

Coming to the Raman experiments on $\mathrm{YBaCuO}$ in the literature, a thorough study is reported by Limonov et al. in Ref. [27], albeit limited to samples in a narrow composition region around optimal doping, with $T_{c} \geqslant 80 \mathrm{~K}$. Their attention is focused on a mode at $\simeq 340 \mathrm{~cm}^{-1}$, that among all Raman-active $A_{1 g}$ modes (in the orthorhombic $D_{2 h}$ classification) stands out for its larger distinct temperature dependence below $T_{c}$. In fact, a softening of over $2 \%$ is shown for overdoped $\left(T_{c}=86 \mathrm{~K}\right)$ and optimally doped $\left(T_{c}=93 \mathrm{~K}\right)$ samples, but much smaller for underdoped $\left(T_{c}=80 \mathrm{~K}\right)$ ones. Their analysis is based on the theory of the phonon self-energies in a strong-coupling superconductor as put forward by Zeyher and Zwicknagl [14], modified for the anisotropic $d$-wave case by Nicol et al. [15]. All other modes have much smaller frequency shifts (positive or negative) at and below $T_{c}$. According to the letter of the analysis of Zeyher and Zwicknagl, the mode is much more affected because in the tetragonal $D_{2 h}$ point group classification this mode would be an "allowed" $B_{1 g}$ mode, while the other considered modes are "forbidden" $A_{1 g}$ (forbidden in the sense that selection rules would impose vanishing values of the matrix elements for the electronphonon interactions affecting the phonon self-energy). This takes the point of view that the orthorhombic distortions are very small, and disorder in the samples essentially washes out their effects, so that tetragonal concepts still apply. A further point to stress is that while the arguments of the present paper predict a sudden drop of the frequency at $T_{c}$, the observed temperature behavior is rather steep but not really sudden. Finally, the mode in question is also not directly identifiable with the ones singled out in Refs. [7,12,13].
So one cannot find support for the nonlinear phononics explanation of light-induced superconductivity in $\mathrm{YBaCuO}$ in a comparison of the criteria discussed here with experiments. It is worthwhile emphasizing that this result does not automatically rule out this explanation, as it could of course, among other possibilities, correspond to the fact that the parameter $c$ describing the effect of the relevant lattice coordinate on $T_{c}$ in the small $Q$ regime happens to be very small $\left(c<0.2 c_{\max }\right)$, or that the form of the Ginzburg Landau description adopted here is oversimplified.

Interestingly, experiments on cuprates with more than two layers, in particular, $\mathrm{HgBa}_{2} \mathrm{Ca}_{3} \mathrm{Cu}_{4} \mathrm{O}_{10+x}$ [28], a compound in which light-enhanced superconductivity is, as far as we know, yet to be looked for, are in qualitative agreement with the predictions of the present analysis. For two modes of $A_{1 g}$ symmetry in this tetragonal structure, with frequencies near 250 and $390 \mathrm{~cm}^{-1}$, respectively, very large softenings (respectively $6 \%$ and $18 \%$, well beyond what the standard self-energy effect of the gap opening would predict) are observed, and they occur rather abruptly in a $10-15 \mathrm{~K}$ interval below $T_{c} \simeq$ $120 \mathrm{~K}$. The interpretation of these experiments is still controversial. According to Munzar and Cardona [29], the unusually large phonon anomalies are related to the appearance, below $T_{c}$, in three- and four-layer cuprates, of a Raman-active transverse Josephson plasma resonance; the charge fluctuations in the cuprate layers induced by the plasmon interact strongly with the charge distortions induced by the phonons, leading to a strong level repulsion and transfer of oscillator strength, with a corresponding enhancement of the Raman intensity for the softened phonon. Indeed, similar phonon anomalies are observed not only in the four-layer $\mathrm{HgBa}_{2} \mathrm{Ca}_{3} \mathrm{Cu}_{4} \mathrm{O}_{10+x}$ but also in the three-layer $\mathrm{Bi}_{2} \mathrm{Si}_{2} \mathrm{Ca}_{2} \mathrm{Cu}_{3} \mathrm{O}_{10+x}$ system [30] with an $8 \%$ softening of a Raman-active phonon at $390 \mathrm{~cm}^{-1}$. However, Limonov et al. observed that the intensity enhancement disappears when the incoming photon energy is increased from $\simeq 1.9 \mathrm{eV}$ to a $2.2-2.5 \mathrm{eV}$ range, suggesting a resonance effect as the explanation. Following a theoretical suggestion by Venturini et al. [31] that a Raman-active $A_{1 g}$ mode arises from collective spin excitations, Gallais et al. [32] compare Raman spectra for the single-layer $\mathrm{HgBa}_{2} \mathrm{CuO}_{4+x}$ and the three-layer $\mathrm{HgBa}_{2} \mathrm{Ca}_{2} \mathrm{Cu}_{3} \mathrm{O}_{8+x}$, and conclude that resonance behavior and scaling with $T_{c}$ of the $A_{1 g}$ modes are rather independent of the number of layers, and therefore do not support the Josephson plasmon explanation.

As far as $\mathrm{Bi}_{2} \mathrm{Si}_{2} \mathrm{Ca}_{2} \mathrm{Cu}_{3} \mathrm{O}_{10+x}$ is concerned, on the other hand, $\mathrm{Hu}$ et al. [33] report the lack of any signatures of light-enhanced critical temperature in this system. Therefore, whatever the reason for the phonon anomaly reported by Limonov et al., it is not related to the mechanism discussed here.

\section{CONCLUSIONS}

In this paper an attempt was made to explore possible experimentally verifiable consequences of the nonlinear phononics interpretation of the phenomenon of light-induced superconductivity. On the basis of a simple Ginzburg-Landau free-energy description, an abrupt softening below $T_{c}$ of one (or more) Raman-active phonon(s) was predicted. Inserting reasonable values for YBCO, it was concluded that the effect 
could be observable in this material. A comparison with existing experiments cannot be claimed to support this prediction, at least for the phonons that are identified as directly related to the $T_{c}$ enhancement.

The observed unusually large and abrupt phonon softenings in $\mathrm{Bi}$ (2223) and $\mathrm{Hg}$ (1234) cuprates with three or four $\mathrm{Cu}-\mathrm{O}$ layers are, on the other hand, qualitatively similar to those predicted here. However, a recent search for lightinduced superconductivity in $\mathrm{Bi}$ (2223) gave a negative result [33], while no search was carried out in $\mathrm{Hg}$ (1234).

On the basis of the presently existing data, the arguments presented here do not lend support to the nonlinear phononics explanation. It may be of interest to explore the observability of a light-induced enhancement of $T_{c}$ in $\mathrm{Hg}$ (1234) and in general to extend the search for the specific phonon modes driving this spectacular phenomenon.

\section{ACKNOWLEDGMENTS}

I am greatly indebted to Andrea Cavalleri for his continuing interest in this work and for many valuable discussions. I am also grateful to Michael Fechner, Michael Först, Andrew J. Millis, Angel Rubio, and Michael Sentef for useful discussions.
[1] D. Fausti, R. I. Tobey, N. Dean, S. Kaiser, A. Dienst, M. C. Hoffmann, S. Pyon, T. Takayama, H. Takagi, and A. Cavalleri, Science 331, 189 (2011).

[2] W. Hu, S. Kaiser, D. Nicoletti, C. R. Hunt, I. Gierz, M. C. Hoffmann, M. Le Tacon, T. Loew, B. Keimer, and A. Cavalleri, Nat. Mater. 13, 705 (2014).

[3] S. Kaiser, C. R. Hunt, D. Nicoletti, W. Hu, I. Gierz, H. Y. Liu, M. Le Tacon, T. Loew, D. Haug, B. Keimer, and A. Cavalleri, Phys. Rev. B 89, 184516 (2014).

[4] M. Mitrano, A. Cantaluppi, D. Nicoletti, S. Kaiser, A. Perucchi, S. Lupi, P. Di Pietro, D. Pontiroli, M. Riccò, S. R. Clark, D. Jaksch, and A. Cavalleri, Nature (London) 530, 461 (2016).

[5] M. Först, C. Manzoni, S. Kaiser, Y. Tomioka, Y. Tokura, R. Merlin, and A. Cavalleri, Nat. Phys. 7, 854 (2011).

[6] A. Subedi, A. Cavalleri, and A. Georges, Phys. Rev. B 89, 220301(R) (2014).

[7] R. Mankowsky et al., Nature 516, 71 (2014).

[8] B. Barbanis, Astron. J. 71, 415 (1966).

[9] K. S. J. Nordholm and S. A. Rice, J. Chem. Phys. 61, 203 (1974); 61, 768 (1974).

[10] F. M. Fernandez, G. A. Arteca, S. A. Maluendes, H. O. Villar, and E. A. Castro, Rev. Bras. Fis. 11, 1053 (1981).

[11] S. Maluendes, G. Arteca, F. M. Fernández, and E. A. Castro, Mol. Phys. 45, 511 (1982); note a misprint in this article in Eq. (8), where the right-hand side should read $\left(n_{2}+1 / 2\right) / w_{2}$.

[12] M. Fechner and N. A. Spaldin, Phys. Rev. B 94, 134307 (2016).

[13] B. Liu, M. Först, M. Fechner, D. Nicoletti, J. Porras, T. Loew, B. Keimer, and A. Cavalleri, Phys. Rev. X, 10, 011053 (2020), including Supplemental Material Sec. S10.

[14] R. Zeyher and G. Zwicknagl, Z. Phys. B: Condens. Matter 78, 175 (1990).

[15] E. J. Nicol, C. Jiang, and J. P. Carbotte, Phys. Rev. B 47, 8131 (1993).
[16] M. Knap, M. Babadi, G. Refael, I. Martin, and E. Demler, Phys. Rev. B 94, 214504 (2016).

[17] E. M. Lifshitz and L. P. Pitaevskii, Statistical Physics, Part 2 , Course of Theoretical Physics Vol. 9 (Pergamon, Oxford, UK, 1980), Sec. 45. (The reader is warned to pay attention to slightly different notations and definitions of the parameters entering the free-energy functional in this textbook, with respect to those adopted here.)

[18] R. Joynt and T. M. Rice, Phys. Rev. B 32, 6074 (1985).

[19] M.-A. Ozaki, Prog. Theor. Phys. 76, 1008 (1986).

[20] A. J. Millis and K. M. Rabe, Phys. Rev. B 38, 8908 (1988).

[21] M. Sigrist and K. Ueda, Rev. Mod. Phys. 63, 239 (1991).

[22] F. Peronaci, M. Schiró, and M. Capone, Phys. Rev. Lett 115 257001 (2015).

[23] C. Calestani and C. Rizzoli, Nature (London) 328, 606 (1987).

[24] J. W. Loram, K. A. Mirza, J. R. Cooper, W. Y. Liang, and J. M. Wade, J. Supercond. 7, 243 (1994).

[25] H. W. Wühl et al., Physica C 185-189, 755 (1991).

[26] A. Knizhnik, G. E. Shter, G. S. Grader, G. M. Reisner, and Y. Eckstein, Physica C 400, 25 (2003).

[27] M. F. Limonov, A. I. Rykov, S. Tajima, and A. Yamanaka, Phys. Rev. B 61, 12412 (2000).

[28] V. G. Hadjiev, X. Zhou, T. Strohm, M. Cardona, Q. M. Lin, and C. W. Chu, Phys. Rev. B 58, 1043 (1998).

[29] D. Munzar and M. Cardona, Phys. Rev. Lett. 90, 077001 (2003).

[30] M. Limonov, S. Lee, S. Tajima, and A. Yamanaka, Phys. Rev. B 66, 054509 (2002).

[31] F. Venturini, U. Michelucci, T. P. Devereaux, and A. P. Kampf, Phys. Rev. B 62, 15204 (2000).

[32] Y. Gallais, A. Sacuto, and D. Colson, Physica C 408-410, 785 (2004).

[33] W. Hu, D. Nicoletti, A. V. Boris, B. Keimer, and A. Cavalleri, Phys. Rev. B 95, 104508 (2017). 